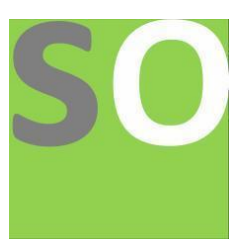

Article title: Power Series.Orthogonal Operational Expansions

Authors: Pablo Enrique Aballe Vazquez[1]

Affiliations: Independent researcher, retired[1]

Orcid ids: 0000-0001-7189-5452[1]

Contact e-mail: aballepe61@gmail.com

License information: This work has been published open access under Creative Commons Attribution License $\mathrm{http}: / / c r e a t i v e c o m m o n s . o r g / l i c e n s e s / b y / 4.0 /$, which permits unrestricted use, distribution, and reproduction in any medium, provided the original work is properly cited. Conditions, terms of use and publishing policy can be found at https://www.scienceopen.com/.

Preprint statement: This article is a preprint and has not been peer-reviewed, under consideration and submitted to ScienceOpen Preprints for open peer review.

DOI: 10.14293/S2199-1006.1.SOR-.PP5HHW9.v1

Preprint first posted online: 11 October 2020

Keywords: Taylor serie, operational orthogonality, differential operator , operational expansions, orthogonal operators, Laplace inversion, operational coefficient 


\title{
Power Series.Orthogonal Operational Expansions
}

\begin{abstract}
Formulation of the classic Taylor series as an orthogonal concept based on identifying the expansion's coefficients as differential transformation applied to a unique function; definition of operational orthogonality by analogy with the Hilbert space and identification of the nth derivative at a point based on the improper integral on the positive semiaxis ; deduction of inversion integrals for Laplacen transforms for analytical functions.
\end{abstract}

\section{Email: aballemat@gmail.com}

\section{I. - Introduction.}

In classical analysis, the nexus between analytical and orthogonal functions is well established with outstanding results for the various fields of application; Thus, the Taylor and Fourier series identify the coefficients starting from the different concepts, the first as derivatives at a point, the second as integrals in an interval. There is no orthogonal character for the system of potential functions.

In this work we establish quantitative relationships for the power series from the classic Laplace and Fourier transforms applying differential operations. On this basis a set of properties are directly deduced from the transforms generation of binomials by action of differential operators acting on a basic primary function, exact identity of the orthogonality operation with the concept of operational orthogonality and the corresponding coefficients, identification of the operational coefficients with the Taylorians. Thus, the derivative at a point, a local property, acquires global character as integral in the positive semiaxis. As a culmination we obtain inversion formulas for the Laplace a transforms starting from the established relations.

We formulate the definition of integrable operational square space in analogy with Hilbert's theory of spaces as a generalization of the concrete examples presented.These concepts are illustrated with two specific examples; on the one hand, the system derived from the generalized Laguerre polynomials requires knowing the analytical behavior at the origin and infinity respectively to calculate the Taylor coefficients at the unit point and for the system derived from the Hermite polynomials, knowing the convolution with the Gaussian it is equivalent to analytic behavior at the origin and infinity as well.

\section{1-Operational orthogonal equivalence. Introductory example.}

In this section we make use of the results of the operational calculation and the orthogonal polynomial theory applied to Laguerre's generalized ones without demonstrating the corresponding theorems, we rely on the extensive bibliography available in this regard.

For what follows we relate certain properties of the Laguerre polynomials and the Laplace transform acting together. Due to their broad reference in the literature and / or elemental character we only indicate them.

- Differentiation operator.

$$
D=\frac{\mathbf{d}}{\mathbf{d t}}
$$

- Generatring function .

$$
\Psi_{\lambda}(\tau, \mathbf{t})=\left[\mathbf{e}^{(-\tau \mathbf{t}) /(1-\tau)}\right] /(\mathbf{1}-\tau)^{\lambda+1}=\sum_{0}^{\infty} \tau^{\mathbf{n}} \mathbf{L}_{\mathbf{n}}^{\lambda}(\mathbf{t})
$$

- Laguerre's differential formula for generalized polynomials.

$\mathbf{L}_{\mathbf{n}}^{\lambda}(\mathbf{t})=\left[\left(\mathbf{e}^{\mathbf{t}} \mathbf{t}^{-\lambda}\right) / \mathbf{n} !\right]\left[\mathbf{D}^{\mathbf{n}}\left(\mathbf{t}^{\lambda+\mathbf{n}} \mathbf{e}^{-\mathbf{t}}\right)\right]$

- Laplace transform.

$\mathbf{L}_{\mathbf{p}}[\mathbf{f}(\mathbf{z})]=\mathbf{F}(\mathbf{p})=\int_{0}^{\infty} \mathrm{e}^{-\mathbf{p t}} \mathbf{f}(\mathbf{t}) \mathbf{d} \mathbf{t}$

-Transform's integration .

$\int_{0}^{\infty} \mathbf{L}_{\mathbf{p}}[\mathbf{f}(\mathbf{z})] \mathbf{d p}=\int_{0}^{\infty} \mathbf{F}(\mathbf{p}) \mathbf{d p}=\int_{0}^{\infty} \mathrm{e}^{-\mathbf{p t}} \frac{\mathbf{f}(\mathbf{t})}{\mathbf{t}} \mathbf{d t}$

- Laguerre generalized polynomial transform .

$\mathbf{L}_{\mathbf{p}}\left[\mathbf{z}^{\lambda} \mathbf{L}_{\mathbf{n}}^{\lambda}(\mathbf{z})\right]=\frac{\Gamma(\mathbf{n}+\lambda+\mathbf{1})}{\Gamma(\mathbf{n}+\mathbf{1}) \mathbf{p}^{\lambda+1}}\left(\mathbf{1}-\frac{\mathbf{1}}{\mathbf{p}}\right)^{\mathbf{n}}, \Gamma(\mathbf{z})$ : función gamma

- Polynomial differential operator action on Laplace transform. 
$\left[\mathbf{P}_{\mathbf{n}}(-\mathbf{D})[\mathbf{F}(\mathbf{p})]\right]_{\mathrm{p}=\mathrm{a}}=\int_{0}^{\infty} \mathbf{P}_{\mathbf{n}}(\mathbf{t}) \mathbf{e}^{-\mathbf{a t}} \mathbf{f}(\mathbf{t}) \mathbf{d t}$

- In particular for Laguerre polynomials .

$\left[\mathbf{L}_{\mathbf{n}}^{\lambda}(-\mathbf{D})[\mathbf{F}(\mathbf{p})]\right]_{\mathbf{p}=\mathbf{a}}=\int_{0}^{\infty} \mathbf{L}_{\mathbf{n}}^{\lambda}(\mathbf{t}) \mathbf{e}^{-\mathbf{a t}} \mathbf{f}(\mathbf{t}) \mathbf{d} \mathbf{t}$

-Operational implications.

$\mathbf{f}(\mathbf{t}) \rightarrow \mathbf{F}(\mathbf{p}) ; \mathrm{e}^{-\mathbf{t}} \mathbf{f}(\mathbf{t}) \rightarrow \mathbf{F}(\mathbf{p}+1) ; \mathbf{t} \mathbf{f}(\mathbf{t}) \mathrm{e}^{-\mathbf{t}} \rightarrow-\mathbf{D F}[(\mathbf{p}+1)]$

$\mathbf{t} \mathbf{L}_{\mathbf{n}}^{\mathbf{0}}(\mathbf{t}) \mathbf{e}^{-\mathbf{t}} \mathbf{f}(\mathbf{t}) \rightarrow-\left[\mathbf{D L}_{\mathbf{n}}(-\mathbf{D})\right][\mathbf{F}(\mathbf{p}+\mathbf{1})]$

- Power generation operation.

$\mathbf{L}_{\mathbf{n}}^{0}(-\mathbf{D})[1 /(\mathbf{p}+1)]=(1-1 /(\mathbf{p}+1))^{\mathbf{n}} /(\mathbf{p}+1)=\int_{0}^{\infty} \mathbf{L}_{\mathbf{n}}(\mathbf{t}) \mathbf{e}^{-(\mathbf{p}+1) \mathbf{t}} \mathbf{d t}$

-Equality of coefficients

$\int_{0}^{\infty} \mathbf{L}_{\mathbf{n}}^{0}(\mathbf{t}) \mathbf{e}^{-\mathbf{t}} \mathbf{f}(\mathbf{t}) \mathbf{d t}=\int_{0}^{\infty}\left[\mathbf{L}_{\mathbf{n}}^{0}(-\mathbf{D})\right][\mathbf{F}(\mathbf{p}+1)] \mathbf{d p}$

-Operational orthogonality.

$\int_{0}^{\infty} \mathbf{L}_{\mathbf{n}}^{0}(\mathbf{t}) \mathbf{L}_{\mathbf{n}}^{0}(\mathbf{t}) \mathbf{e}^{-\mathbf{t}} \mathbf{f}(\mathbf{t}) \mathbf{d t}=-\int_{0}^{\infty}\left[(\mathbf{D}) \mathbf{L}_{\mathbf{m}}^{0}(-\mathbf{D}) \mathbf{L}_{\mathbf{n}}^{0}(-\mathbf{D})\right][1 /(\mathbf{p}+1)] \mathbf{d p}=\left\{\begin{array}{ll}1 & \mathbf{s i m}=\mathbf{n} \\ 0 & \mathbf{s i m} \neq \mathbf{n}\end{array}\right\}$

Let us determine the coefficients for the Laguerre and power series with the previous correlations.

Given the expansions :

$\mathbf{e}^{-\mathbf{z}} \mathbf{f}(\mathbf{z})=\mathbf{e}^{-\mathbf{z}} \sum_{0}^{\infty} \mathbf{a}_{\mathbf{n}} \mathbf{L}_{\mathbf{n}}(\mathbf{z}) ; \mathbf{a}_{\mathbf{n}}=\int_{0}^{\infty} \mathbf{L}_{\mathbf{n}}^{\mathbf{0}}(\mathbf{t}) \mathbf{e}^{-\mathbf{t}} \mathbf{f}(\mathbf{t}) \mathbf{d t}$

The Laplace transformation makes it:

$\mathbf{F}(\mathbf{p}+1)=\sum_{0}^{\infty} \mathbf{a}_{\mathbf{n}}(1-1 /(\mathbf{p}+1))^{\mathbf{n}} /(\mathbf{p}+1)$

\subsubsection{Operational orthogonality and norm for generalized Laguerre polynomials.}

This relationship establishes a strict equality of the norm in the 'original 'space and the operational norm in the 'transformed space and indicates a concept to generalize to arbitrary spaces. Its deduction is direct based on the properties established in section 1.1 .

Be given.:

$\int_{0}^{\infty} \mathbf{t}^{\lambda+1} \mathbf{e}^{-(\mathbf{p}+\mathbf{1}) \mathbf{t}} \mathbf{d t}=-[(\mathbf{D})]\left[\Gamma(\lambda+\mathbf{1}) /(\mathbf{p}+\mathbf{1})^{\lambda+1}\right] ; \lambda>-\mathbf{1}$

Apply the operators $\quad \mathbf{L}_{\mathbf{n}}^{\lambda}(-\mathbf{D}) \quad$ y $\quad \mathbf{L}_{\mathbf{m}}^{\lambda}(-\mathbf{D})$ in any order according to (1.8). Using (1.5) we have:

$$
\int_{0}^{\infty} \mathbf{t}^{\lambda} \mathbf{L}_{\mathbf{m}}^{\lambda}(\mathbf{t}) \mathbf{L}_{\mathbf{n}}^{\lambda}(\mathbf{t}) \mathbf{e}^{-\mathbf{t}} \mathbf{d t}=-\int_{0}^{\infty}\left[(\mathbf{D}) \mathbf{L}_{\mathbf{m}}^{\lambda}(-\mathbf{D}) \mathbf{L}_{\mathbf{n}}^{\lambda}(-\mathbf{D})\right]\left[\Gamma(\lambda+\mathbf{1}) /(\mathbf{p}+\mathbf{1})^{\lambda+1}\right] \mathbf{d p}=\left\{\begin{array}{ll}
\frac{\Gamma(\mathbf{n}+\lambda+\mathbf{1})}{\mathbf{n} !} & \text { si } \mathbf{m}=\mathbf{n} \\
\mathbf{0} & \text { si } \mathbf{m} \neq \mathbf{n}
\end{array}\right\}
$$

The identity allows the calculation of the coefficients of the orthogonal development through operations on the transform.For null order polynomials it looks like .

$$
\int_{0}^{\infty} \mathbf{L}_{\mathbf{n}}^{0}(\mathbf{t}) \mathbf{L}_{\mathbf{n}}^{0}(\mathbf{t}) \mathbf{e}^{-\mathbf{t}} \mathbf{f}(\mathbf{t}) \mathbf{d t}=-\int_{0}^{\infty}\left[(\mathbf{D}) \mathbf{L}_{\mathbf{m}}^{0}(-\mathbf{D}) \mathbf{L}_{\mathbf{n}}^{0}(-\mathbf{D})\right][1 /(\mathbf{p}+1)] \mathbf{d p}=\left\{\begin{array}{ll}
1 & \text { sim }=\mathbf{n} \\
0 & \text { sim } \neq \mathbf{n}
\end{array}\right\}
$$

\subsection{Identity orthogonal and Taylor coefficients.}

Let us determine the coefficients for the null order Laguerre's polynomials and power series with the above correlations.

Let the expansions be given:

$\mathbf{e}^{-\mathbf{z}} \mathbf{f}(\mathbf{z})=\mathbf{e}^{-\mathbf{z}} \sum_{0}^{\infty} \mathbf{a}_{\mathbf{n}} \mathbf{L}_{\mathbf{n}}^{\mathbf{0}}(\mathbf{z}) ; \mathbf{a}_{\mathbf{n}}=\int_{0}^{\infty} \mathbf{L}_{\mathbf{n}}^{\mathbf{0}}(\mathbf{t}) \mathbf{e}^{-\mathbf{t}} \mathbf{f}(\mathbf{t}) \mathbf{d t}$ 
The Laplace transformation makes it :

$$
\mathbf{F}(\mathbf{p}+1)=\sum_{0}^{\infty} \mathbf{a}_{\mathbf{n}}(1-1 /(\mathbf{p}+1))^{\mathbf{n}} /(\mathbf{p}+1)
$$

Note the transformation of Laguerre's system of polynomials into a system of inverse powers of binomial displaced by unity, corresponding to each polynomial an element of the operational space.

With (1.11) it acquires the form.

$$
\mathbf{F}(\mathbf{p}+1)=\sum_{0}^{\infty} \mathbf{a}_{\mathbf{n}} \mathbf{L}_{\mathbf{n}}^{0}(-\mathbf{D})[1 /(\mathbf{p}+1)] \quad(1.21)
$$

Within the summation sign we identify the Laguerre series expansion for the function (1.14) .

$$
\mathbf{F}(\mathbf{p}+1)=\mathbf{f}(-\mathbf{D})[1 /(\mathbf{p}+1)] \quad(1.15)
$$

Each coefficient is determined by application of the property (1.17) with $\lambda=0$.

$$
\mathbf{a}_{\mathbf{n}}=-\int_{0}^{\infty}(\mathbf{D})\left[\left(\mathbf{L}_{\mathbf{n}}^{\mathbf{0}}(-\mathbf{D})\right)\right][\mathbf{F}(\mathbf{p}+\mathbf{1})] \mathbf{d p}=\left.\left[\mathbf{L}_{\mathbf{n}}(-\mathbf{D})\right][\mathbf{F}(\mathbf{p}+\mathbf{1})]\right|_{\mathbf{p}=\mathbf{0}}-\left.\left[\mathbf{L}_{\mathbf{n}}(-\mathbf{D})\right][\mathbf{F}(\mathbf{p}+\mathbf{1})]\right|_{\mathbf{p}=\infty}
$$

The operator must be understood as its representation by the orthogonal series; their action generates a formal power series. The previous relations do not guarantee the convergence of the series of powers, for polynomials the result is exact in view of the expression of an arbitrary polynomial in terms of the Laguerre polynomials. It is interesting to express the transforms as a single function transformation. Next, let's analyze the question of the conditions for the coefficients of 1.14 to be those of Taylor.

We can represent 1.14 in power series in the usual way with an elementary change of variable $z=1 /(p+1)$.

$$
\mathbf{F}(1 / \mathbf{z}) / \mathbf{z}=\sum_{0}^{\infty} \overline{\mathbf{a}_{\mathbf{n}}}(1-\mathbf{z})^{\mathbf{n}}
$$

If (1.14) is Taylor, its coefficients are determined by :

$$
\overline{\mathbf{a}}_{\mathbf{n}}=(-\mathbf{1})^{\mathbf{n}}[\mathbf{F}(\mathbf{1} / \mathbf{z}) / \mathbf{z}]^{\mathbf{n}} / \mathbf{n} ! ; \mathbf{z}=\mathbf{1} \quad(1.24)
$$

Let us demonstrate by direct calculation the identity of these coefficients with those calculated by the previous formulas. The derivation of the result requires the integral representation of $\mathbf{F}(1 / \mathbf{z}) / \mathbf{z}$ in term of the Laplace integral plus the nchasm derivation and comparison with the orthogonal ones (1.14); and the acceptance of the derivability conditions for dependent - parameter integral. In the following the symbol (n) indicates order $\mathrm{n}$ derivatives.

$$
\begin{aligned}
& \mathbf{F}(1 / \mathbf{z}) / \mathbf{z}=(1 / \mathbf{z}) \int_{0}^{\infty} \mathrm{e}^{(-1 / \mathbf{z}) \mathbf{t}} \mathbf{f}(\mathbf{t}) \mathbf{d t} \quad(1.24 .0)
\end{aligned}
$$

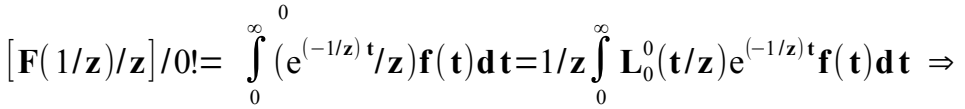

$$
\begin{aligned}
& \overline{\mathbf{a}_{0}}=[[\mathbf{F}(1 / \mathbf{z}) / \mathbf{z}] / 0 !]_{\mathbf{z}=1}=\int_{0}^{\infty} \mathbf{L}_{0}^{0}(\mathbf{t}) \mathrm{e}^{-\mathbf{t}} \mathbf{f}(\mathbf{t}) \mathbf{d} \mathbf{t}=\mathbf{a}_{0} \\
& {[\mathbf{F}(1 / z) / z]^{(1)} / 1 !=(1 / !) \int_{0}^{\infty}\left[\left(\mathbf{e}^{(-1 / z)}\right) / z\right]^{(1)} \mathbf{f}(\mathbf{t}) \mathbf{d} \mathbf{t}=-\left(1 / \mathbf{z}^{2}\right) \int_{0}^{\infty} \mathbf{L}_{1}^{0}(\mathbf{t} / \mathbf{z}) \mathbf{e}^{-(1 / \mathbf{z}) \mathbf{t}} \mathbf{f}(\mathbf{t}) \mathbf{d t} \Rightarrow} \\
& \overline{\mathbf{a}_{1}}=\left[[\mathbf{F}(\mathbf{1} / \mathbf{z}) / \mathbf{z}]^{(\mathbf{1})} / \mathbf{1} !\right]_{\mathrm{z}=1}=\int_{0}^{\infty}[-\mathbf{1}+\mathbf{t}] \mathrm{e}^{-\mathbf{t}} \mathbf{f}(\mathbf{t}) \mathbf{d} \mathbf{t}=-\int_{0}^{\infty}\left[\mathbf{L}_{1}^{0}(\mathbf{t})\right] \mathbf{e}^{-\mathbf{t}} \mathbf{f}(\mathbf{t}) \mathbf{d t}=-\mathbf{a}_{1} \\
& {[\mathbf{F}(1 / \mathbf{z}) / \mathbf{z}]^{(2)} / 2 !=(1 / 2 !) \int_{0}^{\infty}\left[\left(\mathbf{e}^{(-1 / z)}\right) / z\right]^{2} \mathbf{f}(\mathbf{t}) \mathbf{d t}=\left(1 / \mathbf{z}^{3}\right) \int_{0}^{\infty}\left[\mathbf{L}_{2}^{0}(\mathbf{t} / \mathbf{z})\right] \mathbf{e}^{(-1 / \mathbf{z}) \mathbf{t}} \mathbf{f}(\mathbf{t}) \mathbf{d} \mathbf{t} \Rightarrow} \\
& a_{2}=\left[[F(1 / z) / z]^{2} / 2 !\right]_{z=1}=1 / 2 ! \int_{0}^{\infty}\left[2-4 t+t^{2}\right] e^{-t} f(t) d t=\int_{0}^{\infty}\left[L_{2}^{0}(t)\right] e^{-t} f(t) d t=a_{2}
\end{aligned}
$$

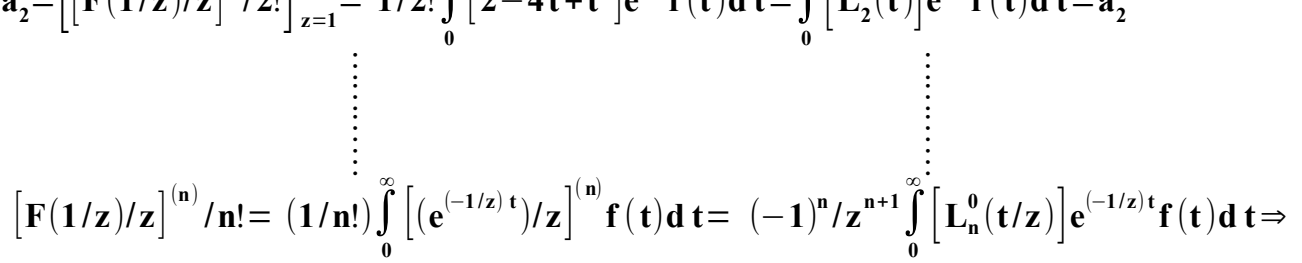

$$
\begin{aligned}
& \overline{\mathbf{a}}_{\mathbf{n}}=[\mathbf{F}(\mathbf{1} / \mathbf{z}) / \mathbf{z}]^{(\mathbf{n})} /\left.\mathbf{n} !\right|_{\mathbf{z}=\mathbf{1}}=\left.(\mathbf{1} / \mathbf{n} !) \int_{0}^{\infty}\left[\left(\mathbf{e}^{(-1 / \mathbf{z}) \mathbf{t}}\right) / \mathbf{z}\right]^{(\mathbf{n})} \mathbf{f}(\mathbf{t}) \mathbf{d} \mathbf{t}\right|_{\mathbf{z}=\mathbf{1}}=(-\mathbf{1})^{\mathbf{n}} \int_{0}^{\infty}\left[\mathbf{L}_{\mathbf{n}}^{\mathbf{0}}(\mathbf{t})\right] \mathbf{e}^{-\mathbf{t}} \mathbf{f}(\mathbf{t}) \mathbf{d t} \mathbf{t}=(-\mathbf{1})^{\mathbf{n}} \mathbf{a}_{\mathbf{n}}
\end{aligned}
$$

This result is also demonstrated from the formula for the generating function :

$\lim _{\mathbf{z} \rightarrow 1} \frac{\left[\left(\mathrm{e}^{(-1 / \mathbf{z}) \mathbf{t}}\right) / \mathbf{z}\right]^{(\mathbf{n})}}{\mathbf{n} !}=\lim _{\mathbf{z} \rightarrow 1} \frac{(-1)^{\mathbf{n}}\left[\mathbf{L}_{\mathbf{n}}^{0}(\mathbf{t} / \mathbf{z})\right] \mathrm{e}^{(-1 / \mathbf{z}) \mathbf{t}}}{\mathbf{z}^{\mathbf{n}+1}}=(-1)^{\mathbf{n}} \mathbf{L}_{\mathbf{n}}^{0}(\mathbf{t}) \mathrm{e}^{-\mathbf{t}}$

Let's highlight some basic facts derived from the previous analysis:

Every function is represented by a series of powers in the environment of the unit.

- The potential function's system is generated by the action of Laguerre operators .

Each function is the result of the action on a single element of an operator represented by the Laguerre series.In other terms all power series is generated by differential transformation of the hyperbola.

_The discontinuous and singular operating operators make sense, example: step operator and delta Dirac.

\subsection{Laplace inversion for analytical functions.}


As an application, we deduce the inversion integral for analytical functions. The Cauchy integral for the derivative of analytical functions allows us to represent the coefficients of the orthogonal development explicitly through the contour integral:

$\mathbf{a}_{\mathbf{n}}=\frac{(-\mathbf{1})^{\mathbf{n}}}{(\mathbf{2} \pi \mathbf{i})} \int_{\mathbf{L}} \frac{\mathbf{F}(\mathbf{1} / \rho)}{\rho(\rho-\mathbf{1})^{\mathbf{n}+\mathbf{1}}} \mathbf{d} \rho \quad, \mathrm{L}$ closed contour. (1.26)

Substituting values the series (1.12) takes the form:

$$
\mathrm{f}(\mathrm{z})=\sum_{0}^{\infty} \frac{(-1)^{\mathrm{n}} \mathrm{L}_{\mathrm{n}}^{0}(\mathrm{z})}{(2 \pi \mathrm{i})} \int_{\mathrm{L}} \frac{\mathrm{F}(1 / \rho)}{\rho(\rho-1)^{\mathrm{n}+1}} \mathrm{~d} \rho
$$

Commuting the sum and the integral.

$\mathrm{f}(\mathrm{z})=\sum_{0}^{\infty} \frac{(-1)^{\mathrm{n}}}{(2 \pi \mathrm{i})} \int_{\mathrm{L}} \frac{\mathrm{F}(1 / \rho) \mathrm{L}_{\mathrm{n}}^{0}(\mathrm{z})}{\rho(\rho-1)^{\mathrm{n}+1}} \mathrm{~d} \rho$

Comparing with the development of the generating function the intraintegral term is easily summable with expression:

$\frac{\mathbf{1}}{\rho-\mathbf{1}} \sum_{\mathbf{0}}^{\infty} \frac{(-\mathbf{1})^{\mathbf{n}} \mathbf{L}_{\mathbf{n}}^{\mathbf{0}}(\mathbf{z})}{(\rho-\mathbf{1})^{\mathbf{n}}}=\frac{\mathbf{e}^{\mathbf{z} / \rho}}{\rho}$

In short, the inversion is made by the integral:

$\mathbf{f}(\mathbf{z})=\frac{1}{2 \pi \mathbf{i}} \int_{\mathbf{L}} \frac{\mathrm{e}^{\mathbf{z} / \rho}}{\rho^{2}} \mathrm{~F}(1 / \rho) \mathbf{d} \rho$

\subsection{1- Expansion in arbitrary point. Coefficients as infinite differential operator.}

By elementally manipulating the nucleus of the integral representation we obtain different variants of development. In general we need knowledge of all the values of the derivatives in one point determined by the type of development. Note that the coefficients for the function at a point require an infinite set of values for a given functional relationship involving the derivatives of the evaluated transform at a point different from that given for the function to be developed. Let's see some cases prior to the general formulation and its corresponding operators.

$$
\begin{aligned}
& \mathbf{f}(\mathbf{z})=\frac{1}{2 \pi \mathbf{i}} \int_{\mathbf{L}} \frac{\mathrm{e}^{\mathbf{z} / \rho}}{\rho^{2}} \mathrm{~F}(1 / \rho) \mathbf{d} \rho=\frac{1}{2 \pi \mathbf{i}} \int_{\mathbf{L}} \frac{\mathrm{e}^{(\mathbf{z}-\mathbf{a}+\mathbf{a}) / \rho}}{\rho^{2}} \mathrm{~F}(1 / \rho) \mathbf{d} \rho=\frac{1}{2 \pi \mathbf{i}} \int_{\mathbf{L}} \frac{\mathrm{F}(1 / \rho)}{\rho^{2}}\left(\sum_{0}^{\infty} \frac{(\mathbf{z}-\mathbf{a})^{\mathrm{n}}}{\mathbf{n} ! \rho^{\mathbf{n}}}\right) \mathrm{e}^{\mathrm{a} / \rho} \mathbf{d} \rho=\sum_{0}^{\infty} \overline{\mathbf{a}_{\mathbf{n}}(\mathbf{z}-\mathbf{a})^{\mathbf{n}}} \\
& \overline{\mathrm{a}_{\mathrm{n}}}=[\mathrm{f}(\mathrm{a})]^{(\mathrm{n})} / \mathrm{n} !=\frac{1}{2 \pi \mathrm{in} !} \int_{\mathrm{L}} \frac{\mathrm{F}(1 / \rho)}{\rho^{\mathrm{n}+2}} \mathrm{e}^{\mathrm{a} / \rho} \mathrm{d} \rho=\frac{1}{2 \pi \mathrm{in} !} \int_{\mathrm{L}} \frac{\mathrm{F}(1 / \rho)}{\rho^{\mathrm{n}+2}} \sum_{0}^{\infty} \frac{(\mathrm{a} / \rho)^{\mathrm{k}}}{\mathrm{k} !} \mathrm{d} \rho
\end{aligned}
$$

Developing the sum and identifying derivatives according to the Cauchy integral.

$\overline{\mathrm{a}_{\mathrm{n}}}=\frac{1}{2 \pi \mathrm{in} !} \int_{\mathrm{L}} \mathrm{F}(1 / \rho)\left[\frac{1}{\rho^{\mathrm{n}+2}}+\frac{\mathrm{a}}{\rho^{\mathrm{n}+2}}+\ldots \frac{\mathrm{a}^{\mathrm{k}}}{\mathrm{k} ! \rho^{\mathrm{n}+\mathrm{k}+2}}\right] \mathrm{d} \rho=\frac{\frac{[\mathrm{F}(1 / \rho)]^{(\mathrm{n}+1)}}{(\mathrm{n}+1) !}+\frac{\mathrm{a}[\mathrm{F}(1 / \rho)]^{(\mathrm{n}+2)}}{(\mathrm{n}+2) !}+\left.\ldots \frac{\mathrm{a}_{\mathrm{k}}[\mathrm{F}(1 / \rho)]^{(\mathrm{n}+\mathrm{k}+1)}}{\mathrm{k} !(\mathrm{n}+\mathrm{k}+1) !}\right|_{\rho=0}}{\mathrm{n} !}$

In terms of the differentiation operator, the numerator verifies the operational relationship.

$$
\frac{[\mathbf{F}(1 / \rho)]^{(\mathbf{n}+1)}}{(\mathbf{n}+1) !}+\frac{\mathbf{a}[\mathbf{F}(1 / \rho)]^{(\mathbf{n}+2)}}{(\mathbf{n}+2) !}+\left.\ldots \frac{\mathbf{a}^{\mathbf{k}}[\mathbf{F}(1 / \rho)]^{(\mathbf{n}+\mathbf{k}+1)}}{\mathbf{k} !(\mathbf{n}+\mathbf{k}+1) !}\right|_{\rho=0}=\left.\left[(\mathbf{D} / \mathbf{a})^{(\mathbf{n}+1) / 2} \mathbf{I}_{\mathbf{n}+1}(2 \sqrt{\mathbf{a D}})\right] \mathbf{F}(1 / \rho)\right|_{\rho=0}
$$

This expression of the coefficients implies the evaluation of a differential operator of infinite order at the origin represented by the Bessel function of imaginary argument. Let us highlight the fact of developability at an arbitrary point with the knowledge of the analytical character of the transformed in the origin , additionally the points of development are located in different spaces.

\subsubsection{Expansion in finite interval}

According to $m$ let's expand two functions which depend on arbitrary constants $a$ and $b$

$$
\begin{aligned}
& \mathbf{F}(\mathbf{p}+\mathbf{a}+1)=\sum_{0}^{\infty} \mathbf{a}_{\mathbf{n}}(1-1 /(\mathbf{p}+1))^{\mathbf{n}} /(\mathbf{p}+1) \\
& \mathbf{F}(\mathbf{p}+\mathbf{b}+1)=\sum_{0}^{\infty} \mathbf{b}_{\mathbf{n}}(1-1 /(\mathbf{p}+1))^{\mathbf{n}} /(\mathbf{p}+1)
\end{aligned}
$$

Each coefficient has the following expression for (1.36):

$$
\mathbf{a}_{\mathbf{n}}=-\int_{\mathbf{0}}^{\infty}(\mathbf{D})\left[\left(\mathbf{L}_{\mathbf{n}}^{\mathbf{0}}(-\mathbf{D})\right)\right][\mathbf{F}(\mathbf{p}+\mathbf{a}+\mathbf{1})] \mathbf{d p}
$$

And respectively for (W)

$$
\mathbf{b}_{\mathbf{n}}=-\int_{\mathbf{0}}^{\infty}(\mathbf{D})\left[\left(\mathbf{L}_{\mathbf{n}}^{\mathbf{0}}(-\mathbf{D})\right)\right][\mathbf{F}(\mathbf{p}+\mathbf{b}+\mathbf{1})] \mathbf{d p}
$$

The difference of both functions is determined by the difference of their coefficients defined by the corresponding integrals in which we make the elementary changes of variables $\quad \mathbf{z}=\mathbf{p}+\mathbf{a} \quad \mathbf{y} \quad \mathbf{z}=\mathbf{p}+\mathbf{b}$.

$$
\mathbf{a}_{\mathbf{n}}=-\int_{\mathbf{a}}^{\infty}(\mathbf{D})\left[\left(\mathbf{L}_{\mathbf{n}}^{\mathbf{0}}(-\mathbf{D})\right)\right][\mathbf{F}(\mathbf{z}+\mathbf{1})] \mathbf{d z}
$$




$$
\mathbf{b}_{\mathbf{n}}=-\int_{\mathbf{b}}^{\infty}(\mathbf{D})\left[\left(\mathbf{L}_{\mathbf{n}}^{\mathbf{0}}(-\mathbf{D})\right)\right][\mathbf{F}(\mathbf{z}+\mathbf{1})] \mathbf{d z}
$$

\section{Con esto :}

$$
\begin{aligned}
& \mathbf{F}(\mathbf{p}+\mathbf{a}+1)-\mathbf{F}(\mathbf{p}+\mathbf{b}+1)=\sum_{0}^{\infty} \mathbf{c}_{\mathbf{n}}(1-1 /(\mathbf{p}+1))^{\mathbf{n}} /(\mathbf{p}+1) \\
& \mathbf{c}_{\mathbf{n}}=\mathbf{a}_{\mathbf{n}}-\mathbf{b}_{\mathbf{n}}=-\int_{\mathbf{a}}^{\mathbf{b}}(\mathbf{D})\left[\left(\mathbf{L}_{\mathbf{n}}^{\mathbf{0}}(-\mathbf{D})\right)\right][\mathbf{F}(\mathbf{z}+\mathbf{1})] \mathbf{d z}
\end{aligned}
$$

This expression contradicts the logic of operational orthogonal expansion in the formal sense; the formula suggests a discontinuous function defined in the interval and null in the rest but your meaning is different. Comparing with the Taylor series, it calculates the difference of the coefficients ( $n$th derivative at $z=1$ ) with a unique expression.

$$
\left.\frac{1}{n !}\left[\frac{F(a+1 / z)}{z}\right]^{(n)}\right|_{z=1}-\left.\frac{1}{n !}\left[\frac{F(b+1 / z)}{z}\right]^{(n)}\right|_{z=1}=\int_{a}^{b}(D)\left[\left(L_{n}^{0}(-D)\right)\right][F(z+1)] d z
$$

\section{II.-Operational orthogonality concept.}

\section{1-Definition .}

For the definition we will take some concepts in analogy of the integrable square function space $\mathbf{L}^{2}(\mathbf{a}, \mathbf{b})$.

1.- Scalar product. Let the fixed function $\mathrm{M}(\mathrm{z})$ be given for all operation and $\mathbf{F}(\mathbf{D}), \mathbf{G}(\mathbf{D})$ differentiation operator's functions . Then:

$$
(\mathbf{F}, \mathbf{G})_{\text {op }}=\int_{\mathbf{a}}^{\mathbf{b}}[\mathbf{F}(\mathbf{D}) \mathbf{G}(\mathbf{D})][\mathbf{M}(\mathbf{z})] \mathbf{d z}
$$

2.- Orthogonal property .

If : $\quad(\mathbf{F}, \mathbf{G})_{\mathbf{o p}}=\int_{\mathbf{a}}^{\mathbf{b}}[\mathbf{F}(\mathbf{D}) \mathbf{G}(\mathbf{D})][\mathbf{M}(\mathbf{z})] \mathbf{d z}=0$

Then $\mathbf{F}(\mathbf{D}), \mathbf{G}(\mathbf{D})$ are orthogonal operators.

3.- Norm square.

$$
\|\mathbf{F}\|^{2}=\int_{\mathbf{a}}^{\mathbf{b}}[\mathbf{F}(\mathbf{D})]^{2}[\mathbf{M}(\mathbf{z})] \mathbf{d z}
$$

Generalizing the introductory example let us formulate a definition of operational orthogonality by analogy of the integrable square function space.

Definition: Let us give a system of functions of the differentiation operator $\Psi \mathrm{n}(\mathrm{D})$ con $\mathrm{n}=1,2,3, \ldots$, a function $\mathrm{M}(\mathrm{z})$ that we will call the primary function, an interval $(\mathrm{a}, \mathrm{b})$, a weight function of the differentiation operator and an arbitrary function $\mathrm{f}(\mathrm{z})$; operational series expansions is defined by:

$$
\mathbf{f}(\mathbf{z})=\sum_{0}^{\infty} \mathbf{a}_{\mathbf{n}} \Psi_{\mathbf{n}}(\mathbf{D})[\mathbf{M}(\mathbf{z})]
$$

With the designation.

$$
\mathbf{F}(\mathbf{D})=\sum_{0}^{\infty} \mathbf{a}_{\mathbf{n}} \Psi_{\mathbf{n}}(\mathbf{D})
$$

Then every function is the result of applying a differential operator to a single function

$$
\mathbf{f}(\mathbf{z})=\mathbf{F}(\mathbf{D})[\mathbf{M}(\mathbf{z})]
$$

Let us designate the space thus defined by $\mathbf{L}_{\mathbf{o p}}^{2}(\mathbf{a}, \mathbf{b})$, with the property of representing an arbitrary element as a transformation of a single element, every element is defined by the action of an operator on the primary function The following properties have to be verified:

-Orthogonality condition:

$$
\int_{\mathbf{a}}^{\mathbf{b}}[\Psi \mathbf{m}(\mathbf{D}) \Psi \mathbf{n}(\mathbf{D})][\rho(\mathbf{D})][\mathbf{M}(\mathbf{z})] \mathbf{d z}=\left\{\begin{array}{ll}
\|\Psi \mathbf{n}\|^{2} & \text { si } \mathbf{m}=\mathbf{n} \\
0 & \text { si } \mathbf{m} \neq \mathbf{n}
\end{array}\right\}
$$

-The coefficients are determined by:

$$
\mathbf{a}_{\mathbf{n}}=\frac{\int_{\mathbf{a}}^{\mathbf{b}}[\Psi \mathbf{n}(\mathbf{D})][\rho(\mathbf{D})][\mathbf{f}(\mathbf{z})] \mathbf{d z}}{\|\Psi \mathbf{n}\|^{2}}
$$

\section{1- Application to generalized Laguerre polynomials.}


Extending the introductory example let us extend the operational orthogonal expansion based on Laguerre's generalized orthogonal polynomials. The necessary properties are derived from their relationship with the Laplace transform deduced by pure analogy. Let us now show them without proof.

1.-Serie expansion .

$$
\mathbf{f}(\mathbf{z})=\sum_{\mathbf{0}}^{\infty} \mathbf{a}_{\mathbf{n}} \mathbf{L}_{\mathbf{n}}^{\lambda}(\mathbf{z}) ; \mathbf{a}_{\mathbf{n}}=\frac{\Gamma(\mathbf{n}+\mathbf{1})}{\Gamma(\mathbf{n}+\lambda+\mathbf{1})} \int_{0}^{\infty} \mathbf{t}^{\lambda} \mathbf{L}_{\mathbf{n}}^{\lambda}(\mathbf{t}) \mathbf{e}^{-\mathbf{t}} \mathbf{f}(\mathbf{t}) \mathbf{d t}
$$

2.-Power generation operation.

$$
\mathbf{L}_{\mathbf{n}}^{\lambda}(-\mathbf{D})\left[\left[\Gamma(\lambda+1) /(\mathbf{p}+1)^{\lambda+1}\right]\right]=\frac{\Gamma(\mathbf{n}+\lambda+1)}{\Gamma(\mathbf{n}+1)(\mathbf{p}+1)^{\lambda+1}}\left(1-\frac{1}{\mathbf{p}+1}\right)^{\mathbf{n}}=\int_{0}^{\infty} \mathbf{t}^{\lambda} \mathbf{L}_{\mathbf{n}}^{\lambda}(\mathbf{t}) \mathbf{e}^{-(\mathbf{p}+1) \mathbf{t}} \mathbf{d t}
$$

3.---Laplace transformation of product potential and exponential functions.

$$
\mathrm{L}_{\mathrm{p}}\left[\mathrm{e}^{-\mathrm{t}} \mathrm{t}^{\lambda} \mathrm{f}(\mathrm{t})\right]=\mathrm{L}_{\mathrm{p}}\left[\mathrm{e}^{-\mathrm{t}} \sum_{0}^{\infty} \mathrm{a}_{\mathrm{n}} \mathrm{t}^{\lambda} \mathrm{L}_{\mathrm{n}}^{\lambda}(\mathrm{t})\right]=\sum_{0}^{\infty} \mathrm{a}_{\mathrm{n}} \frac{\Gamma(\mathrm{n}+\lambda+1)}{\Gamma(\mathrm{n}+1)(\mathrm{p}+1)^{\lambda+1}}\left(1-\frac{1}{\mathrm{p}+1}\right)^{\mathrm{n}}
$$

4 .. Operational expression.

$$
\mathbf{L}_{\mathbf{p}}\left[\mathbf{e}^{-\mathbf{t}} \mathbf{t}^{\lambda} \mathbf{f}(\mathbf{t})\right]=\sum_{\mathbf{0}}^{\infty} \mathbf{a}_{\mathbf{n}} \mathbf{L}_{\mathbf{n}}^{\lambda}(-\mathbf{D})\left[\Gamma(\lambda+\mathbf{1}) /(\mathbf{p}+\mathbf{1})^{\lambda+\mathbf{1}}\right]
$$

$\mathrm{L}_{\mathrm{p}}\left[\mathrm{e}^{-\mathrm{t}} \mathrm{t}^{\lambda} \mathrm{f}(\mathrm{t})\right]=\mathrm{f}(-\mathrm{D})\left[\Gamma(\lambda+1) /(\mathrm{p}+1)^{\lambda+1}\right]$

For $\lambda$ positive integer $\lambda=0,1,2,3, \ldots \mathrm{k}$ we have explicit expressions.

$\mathrm{L}_{\mathrm{p}}\left[\mathrm{e}^{-\mathrm{t}} \mathrm{t}^{\mathrm{k}} \mathrm{f}(\mathrm{t})\right]=(-\mathrm{D})^{\mathrm{k}}[\mathrm{F}(\mathrm{p}+1)]=(-1)^{(\mathrm{k})}[\mathrm{F}(\mathrm{p}+1)]^{\mathrm{k}}=\mathrm{f}(-\mathrm{D})\left[\Gamma(\lambda+1) /(\mathrm{p}+1)^{\lambda+1}\right]=(-\mathrm{D})^{\mathrm{k}} \mathrm{f}(-\mathrm{D})[1 /(\mathrm{p}+1)]$

5.- Taylorian coefficient orthogonal coefficient relationship.

With substitution, $\mathbf{1} /(\mathrm{p}+\mathbf{1})=\mathrm{Z}$ the series $(\tilde{\mathrm{N}})$ takes the form.

$$
\frac{\left.\mathrm{L}_{\mathrm{p}}\left[\mathrm{e}^{-\mathrm{t}} \mathrm{t}^{\lambda} \mathrm{f}(\mathrm{t})\right]\right|_{\mathrm{p}=1 / \mathrm{z}-1}}{\mathrm{z}^{\lambda+1}}=\frac{1}{\mathrm{z}^{\lambda+1}} \int_{0}^{\infty} \mathrm{t}^{\lambda} \mathrm{e}^{-\frac{\mathrm{t}}{\mathrm{z}}} \mathrm{f}(\mathrm{t}) \mathrm{d} \mathrm{t}=\sum_{0}^{\infty} \mathrm{a}_{\mathrm{n}} \frac{\Gamma(\mathrm{n}+\lambda+1)}{\Gamma(\mathrm{n}+1)}(1-\mathrm{z})^{\mathrm{n}}
$$

Taylor's conditions require:

$$
\overline{a_{n}}=\left[\frac{\left.L_{p}\left[e^{-t} t^{\lambda} f(t)\right]\right|_{p=1 / z-1}}{z^{\lambda+1}}\right]^{(n)} / n !=(1 / n !) \int_{0}^{\infty}\left[\left(e^{(-1 / z) t}\right) / z^{\lambda+1}\right]^{(n)} t^{\lambda} f(t) d t, z=1
$$

Note that the intraintegral term is a version of the generating function, under a change of variable it has the expansion .

$$
\Psi_{\lambda}(\mathbf{1}-\mathbf{z}, \mathbf{t})=\left[\mathbf{e}^{-(\mathbf{1}-\mathbf{z}) \mathbf{t} /(\mathbf{z})}\right] /(\mathbf{z})^{\lambda+1}=\sum_{\mathbf{0}}^{\infty}(\mathbf{1}-\mathbf{z})^{\mathbf{n}} \mathbf{L}_{\mathbf{n}}^{\lambda}(\mathbf{t})
$$

From where :

$$
\left[\mathbf{e}^{-\mathbf{t} /(\mathbf{z})}\right] /(\mathbf{z})^{\lambda+1}=\mathbf{e}^{-\mathbf{t}} \sum_{\mathbf{0}}^{\infty}(\mathbf{1}-\mathbf{z})^{\mathbf{n}} \mathbf{L}_{\mathbf{n}}^{\lambda}(\mathbf{t})
$$

Comparing with Taylor's development in $\mathrm{z}=1$, we infer:

$\left[\left[\mathrm{e}^{-\mathrm{t} /(\mathrm{z})}\right] /(\mathrm{z})^{\lambda+1}\right]^{(\mathrm{n})} /\left.\mathrm{n} !\right|_{\mathrm{z}=1}=\mathrm{e}^{-\mathrm{t}}(-1)^{\mathrm{n}} \mathrm{L}_{\mathrm{n}}^{\lambda}(\mathrm{t})$

And from here.

$$
\overline{a_{n}}=\frac{\left[\frac{\left.L_{p}\left[e^{-t} t^{\lambda} f(t)\right]\right|_{p=1 / z-1}}{z^{\lambda+1}}\right]^{(n)}}{n !}=\lim _{z \rightarrow 1} \frac{1}{n !} \int_{0}^{\infty}\left[\left(e^{(-1 / z) t}\right) / z^{\lambda+1}\right]^{(n)} t^{\lambda} f(t) d t=(-1)^{n} \int_{0}^{\infty} L_{n}^{\lambda}(t) t^{\lambda} e^{-t} f(t) d t=(-1)^{n} \frac{\Gamma(n+\lambda+1)}{\Gamma(n+1)} a_{n}
$$

6.-Inversion formula.

Making use of the coefficients of the series expansion of powers (2.19) we reduce (2.9) to the form.

$$
\mathbf{f}(\mathbf{z})=\sum_{\mathbf{0}}^{\infty} \mathbf{a}_{\mathbf{n}} \mathbf{L}_{\mathbf{n}}^{\lambda}(\mathbf{z})=\sum_{\mathbf{0}}^{\infty}(-\mathbf{1})^{\mathbf{n}} \overline{\mathbf{a}}_{\mathbf{n}} \frac{\Gamma(\mathbf{n}+\mathbf{1})}{\Gamma(\mathbf{n}+\lambda+\mathbf{1})} \mathbf{L}_{\mathbf{n}}^{\lambda}(\mathbf{z})
$$

Let us express each coefficient through the Cauchy integral. Designating:

$$
\begin{aligned}
& \mathbf{Q}(\mathbf{z})=\left.\mathbf{L}_{\mathbf{p}}\left[\mathrm{e}^{-\mathbf{t}} \mathbf{t}^{\lambda} \mathbf{f}(\mathbf{t})\right]\right|_{\mathbf{p}=1 / \mathbf{z}-1}(2.21) \\
& \overline{\mathrm{a}_{\mathrm{n}}}=\left.\frac{\left[\frac{\mathrm{Q}(\mathrm{z})}{\mathrm{z}^{\lambda+1}}\right]^{(\mathrm{n})}}{\mathrm{n} !}\right|_{\mathrm{z}=1}=\frac{(-1)^{\mathrm{n}}}{(2 \pi \mathrm{i})} \int_{\mathrm{L}} \frac{\mathrm{Q}(\rho)}{\rho^{\lambda+1}(\rho-1)^{\mathrm{n}+1}} \mathrm{~d} \rho
\end{aligned}
$$

From here

$$
\mathbf{f}(\mathbf{z})=\sum_{\mathbf{0}}^{\infty} \mathbf{a}_{\mathbf{n}} \mathbf{L}_{\mathbf{n}}^{\lambda}(\mathbf{z})=\sum_{\mathbf{0}}^{\infty} \frac{\Gamma(\mathbf{n}+\mathbf{1})}{\Gamma(\mathbf{n}+\lambda+\mathbf{1})} \frac{(-\mathbf{1})^{\mathbf{n}}}{(\mathbf{2} \pi \mathbf{i})} \int_{\mathbf{L}} \frac{\mathbf{Q}(\rho) \mathbf{L}_{\mathbf{n}}^{\lambda}(\mathbf{z})}{\rho^{\lambda+\mathbf{1}}(\rho-\mathbf{1})^{\mathbf{n}+1}} \mathbf{d} \rho
$$

To calculate the sum, let us introduce the fractional integration operator, whose action is defined by:

$$
\mathrm{D}^{-\lambda}[\mathrm{f}(\mathrm{y})]=\frac{1}{\Gamma(\lambda)} \int_{0}^{\mathrm{y}}(\mathrm{y}-\mathrm{t})^{\lambda-1} \mathrm{f}(\mathrm{t}) \mathrm{dt} ; \mathrm{D}^{-1}[\mathrm{f}(\mathrm{y})]=\int_{0}^{\mathrm{y}} \mathrm{f}(\mathrm{y}) \mathrm{dy} ; \mathrm{D}^{-\mathrm{n}}[\mathrm{f}(\mathrm{y})]=\underbrace{\int_{0}^{\mathrm{y}} \mathrm{y}}_{\mathrm{n} \text { integrals }} \mathrm{f}(\mathrm{y}) \mathrm{d}^{\mathrm{n}} \mathrm{y} \text { forn } \in \mathbb{N}
$$


In particular .

$$
\mathrm{D}^{-\lambda}\left[\mathrm{y}^{\mathrm{n}}\right]=\frac{\Gamma(\mathrm{n}+1)}{\Gamma(\mathrm{n}+\lambda+1)} \mathrm{y}^{\mathrm{n}+\lambda}
$$

Applying the operator $\left[1 / \mathrm{y}^{\lambda}\right] \mathrm{D}^{-\lambda}$ to the product of the potential function and the generatrix we obtain.

$$
\frac{\mathbf{1}}{\mathbf{y}^{\lambda}} \mathbf{D}^{-\lambda}\left[\mathbf{y}^{\lambda} \Psi_{\lambda}(-\mathbf{y}, \mathbf{z})\right]=\frac{\mathbf{1}}{\mathbf{y}^{\lambda}} \mathbf{D}^{-\lambda}\left[\mathbf{y}^{\lambda}\left[\mathbf{e}^{(\mathbf{y} \mathbf{z}) /(\mathbf{1}+\mathbf{y})}\right] /(\mathbf{1}+\mathbf{y})^{\lambda+\mathbf{1}}\right]=\sum_{\mathbf{0}}^{\infty} \frac{(-\mathbf{1})^{\mathbf{n}}}{\mathbf{y}^{\lambda}} \mathbf{D}^{-\lambda}\left[\mathbf{y}^{\mathbf{n}+\lambda} \mathbf{L}_{\mathbf{n}}^{\lambda}(\mathbf{z})\right]=\sum_{\mathbf{0}}^{\infty} \frac{(-\mathbf{1})^{\mathbf{n}} \Gamma(\mathbf{n}+\mathbf{1})}{\Gamma(\mathbf{n}+\lambda+\mathbf{1})}\left[\mathbf{y}^{\mathbf{n}} \mathbf{L}_{\mathbf{n}}^{\lambda}(\mathbf{z})\right]
$$

Now with the substitution $y=1 /(\rho-1)$ we obtain the sum; even $\lambda=1,2,3 \ldots$ there are no explicit formulas, the integral is not solvable in elementary terms. To simplify the integral representation, let us identify the nucleus by.

$$
\mathrm{K}_{\lambda}(\rho, \mathrm{z})=\left.\frac{1}{\mathrm{y}^{\lambda}} \mathrm{D}^{-\lambda}\left[\mathrm{y}^{\lambda} \Psi_{\lambda}(-\mathrm{y}, \mathrm{z})\right]\right|_{\mathrm{y}=1 /(\rho-1)}
$$

With this (2.23) determines the inversion formula in final form.

$$
\mathrm{f}(\mathrm{z})=\frac{1}{(2 \pi \mathrm{i})} \int_{\mathrm{L}} \frac{\mathrm{K}_{\lambda}(\rho, \mathrm{z}) \mathrm{Q}(\rho)}{\rho^{\lambda+1}(\rho-1)} \mathrm{d} \rho
$$

\section{2- System based on Hermite polynomials.}

Let's build the system based on the relationship of the Hermite polynomials with the Fourier transform. This system is characterized by three basic elements; use of Hermite operational polynomials of imaginary argument; as a primary function the Gaussian and as a weight function the differentiation operator, also as an integration interval the positive number line. In analogy with Laguerre's operational orthogonal basis let us deduce the conditions of orthogonality by equality to certain operations on the transform; specifically :

Let be given the Fourier transform.

$$
\mathbf{L}_{\mathbf{f}}[\mathbf{f}(\mathbf{t})]=\mathbf{F}(\mathbf{z})=\int_{-\infty}^{\infty} \mathrm{e}^{-\mathbf{i} \mathbf{z t}} \mathbf{f}(\mathbf{t}) \mathbf{d t} \quad(2.30)
$$

Let's apply operations on the transform of $\mathrm{f}(\mathrm{t})=\mathrm{e}^{-\mathrm{t}^{2}}$ and deduce the corresponding results :

$$
\begin{aligned}
& \mathbf{L}_{\mathbf{f}}\left[\mathbf{e}^{-\mathbf{t}^{2}}\right]=\sqrt{\pi} \mathbf{e}^{-\mathbf{z}^{2} / 4}=\int_{-\infty}^{\infty} \mathbf{e}^{-\mathbf{i} \mathbf{z} \mathbf{t}} \mathbf{e}^{-\mathbf{t}^{2}} \mathbf{d t} \\
& \mathbf{H}_{\mathbf{n}}(\mathbf{i} \mathbf{D})\left[\sqrt{\pi} \mathrm{e}^{-\mathbf{z}^{2} / 4}\right]=\int_{-\infty}^{\infty} \mathbf{H}_{\mathbf{n}}(\mathbf{t}) \mathrm{e}^{-\mathbf{t}^{2}} \mathrm{e}^{-\mathbf{i} \mathbf{z t}} \mathbf{d t} \quad ; \quad[-\mathbf{D}]\left[\mathbf{H}_{\mathbf{n}}(\mathbf{i} \mathbf{D})\right]\left[\sqrt{\pi} \mathrm{e}^{-\mathbf{z}^{2} / 4}\right]=\int_{-\infty}^{\infty}(\mathbf{i t}) \mathbf{H}_{\mathbf{n}}(\mathbf{t}) \mathrm{e}^{-\mathbf{t}^{2}} \mathbf{e}^{-\mathbf{i z t} \mathbf{d}} \mathbf{d t} \\
& \sqrt{\pi} \int_{0}^{\infty}[-D]\left[\mathbf{H}_{\mathbf{n}}(\mathbf{i D})\right]\left[\mathbf{H}_{\mathrm{m}}(\mathbf{i} \mathbf{D})\right]\left[\mathbf{e}^{-\mathbf{z}^{2} / 4}\right] \mathbf{d z}=\int_{-\infty}^{\infty} \mathbf{H}_{\mathbf{n}}(\mathbf{t}) \mathbf{H}_{\mathbf{m}}(\mathbf{t}) \mathbf{e}^{-\mathbf{z}^{2}} \mathbf{d t}=\left\{\begin{array}{lll}
\sqrt{\pi} \mathbf{2}^{\mathbf{n}} \mathbf{n} ! & \text { si } & \mathbf{n}=\mathbf{m} \\
\mathbf{0} & \text { si } & \mathbf{n} \neq \mathbf{m}
\end{array}\right\}
\end{aligned}
$$

With this proven orthogonality operation we arrive at the expansion theorem.

$$
\mathrm{F}(\mathrm{z})=\sqrt{\pi} \sum_{0}^{\infty} \mathrm{a}_{\mathrm{n}} \mathrm{H}_{\mathrm{n}}(\mathrm{iD})\left[\mathrm{e}^{-\mathrm{z}^{2} / 4}\right] \quad \mathrm{a}_{\mathrm{n}}=\frac{1}{\pi 2^{\mathrm{n}} \mathrm{n} !} \int_{0}^{\infty}[-\mathrm{D}]\left[\mathrm{H}_{\mathrm{n}}(\mathrm{iD})\right][\mathrm{F}(\mathrm{z})] \mathrm{dz}
$$

\subsection{1 -Orthogonal and Taylorian coefficients relationship .}

-Expansion in power series .

Let us transform the Hermite series into a power series with the Fourier transform.

If:

$$
\mathbf{e}^{-\mathbf{t}^{2}} \mathbf{f}(\mathbf{z})=\mathbf{e}^{-\mathbf{t}^{2}} \sum_{\mathbf{0}}^{\infty} \mathbf{a}_{\mathbf{n}} \mathbf{H}_{\mathbf{n}}(\mathbf{t})
$$

Applying Fourier.

$$
\mathbf{L}_{\mathbf{f}}\left[\mathrm{e}^{-\mathbf{t}^{2}} \mathbf{f}(\mathbf{t})\right]=\frac{1}{\sqrt{2 \pi}} \int_{-\infty}^{\infty} \mathrm{e}^{-\mathbf{t}^{2} / 4} \mathbf{L}_{\mathbf{f}}[\mathbf{F}(\mathbf{t}-\mathbf{z})] \mathbf{d t}=\sum_{0}^{\infty} \mathbf{a}_{\mathbf{n}} \mathbf{H}_{\mathbf{n}}(\mathbf{i D})\left[\sqrt{ } \pi \mathrm{e}^{-\mathbf{z}^{2} / 4}\right]=\sum_{0}^{\infty} \mathbf{a}_{\mathbf{n}}(-\mathbf{i} \mathbf{z})^{\mathbf{n}} \mathrm{e}^{-\mathbf{z}^{2} / 4}
$$

-Expansion in Taylor series .

The formula (2.36) indicates obtaining the Taylor series by expanding the function:

$$
\mathrm{e}^{\mathbf{z}^{2}} \mathbf{L}_{\mathbf{f}}\left[\mathrm{e}^{-\mathbf{t}^{2}} \mathbf{f}(\mathbf{t})\right]=\mathrm{e}^{\mathbf{z}^{2}} \int_{-\infty}^{\infty} \mathrm{e}^{-\mathbf{t}^{2}} \mathbf{f}(\mathbf{t}) \mathrm{e}^{-\mathbf{i} \mathbf{z t}} \mathbf{d} \mathbf{t}=\frac{\mathrm{e}^{\mathbf{z}^{2}}}{\sqrt{2 \pi}} \int_{-\infty}^{\infty} \mathrm{e}^{-\mathbf{t}^{2} / 4} \mathbf{F}_{\mathbf{f}}(\mathbf{t}-\mathbf{z}) \mathbf{d t}=\sum_{0}^{\infty} \mathbf{b}_{\mathbf{n}} \mathbf{z}^{\mathbf{n}}
$$

The value of each coefficient is determined by the relation:

$$
\mathbf{b}_{\mathbf{n}}=\frac{(-\mathbf{i})^{\mathbf{n}}}{2^{\mathbf{n}} \mathbf{n} !} \int_{-\infty}^{\infty} \mathbf{H}_{\mathbf{n}}(\mathbf{t}) \mathbf{f}(\mathbf{t}) \mathrm{e}^{-\mathbf{t}^{2}} \mathbf{d t}
$$

-Correlation of coefficients

Comparing with the coefficients of the Hermite series we obtain:

$$
\mathbf{a}_{\mathbf{n}}=\frac{(-\mathbf{i})^{\mathbf{n}} \mathbf{b}_{\mathbf{n}}}{\sqrt{\pi}}
$$

\section{Conclusions.}

1.-identification of power serie's expansions as orthogonal concept

2.-The definition by analogy of the concept of operational orthogonality or space of integrable square operators opens the field to the general investigation of these spaces. 
3.-The formulation of a new operational orthogonal procedure for the series development of powers in the environment of the unit only requires knowledge of the analytical nature of the function at the origin and point of infinity.

4.-Every power serie is the result of the application of a differential operator on a single basic function. With this, all analytical behavior is a consequence of the transformation of a primary phenomenon.

\section{REFERENCES}

[1] Abate J, Choudhury G, Whitt W. On the Laguerre-method for numerically inverting Laplace transforms.

INFORMS Journal of Computing 1996; 8:413-427.

[2] Abate J , and Valko P.P, Multi-precision Laplace transform inversion, Int. J. Numer. Meth.Engng., 60, 5, 979-993, 2004.

[3] Barnaby.N and Kamran.N, Dynamics with infinitely many derivatives: the initial value problem. J. High Energy Physics 2008 no. 02, Paper 008, 40 pp.

[4] Barnaby.N and Kamran-N, Dynamics with infinitely many derivatives: variablecoefficient equations. J. High Energy Physics 2008 no. 12, Paper 022, 27 pp.

[5]Bravo Yuste,Santos, Metodos Matematicos Avanzados Para Cietíficos E Ingenieros ,Publicaciones Univ.Extremadura Publicaciones ,2006

[6] Carmichael ,R.D .Linear differential equations of infinite order-Bull. Amer. Math. Soc., Volume 42, Number 4 (1936), 193-218

[7] Churchill.R.V, Operational Mathematics, McGraw-Hill, Inc , 1958

[ 8]Davis, H.T “The Theory of Linear Operators from the Standpoint of Differential

Equations of Infinite Order,” The Principia Press (1936).

[9] Domanski, P., Langenbruch, M., Hadamard multipliers on spaces of real analytic functions, Adv. Math., 240 (2013), $575-612$.

[10] Domanski, P Notes on real analytic functions and classical operators, in: "Topics in ComplexAnalysis and Operator Theory", Proc. Third Winter School in Complex Analysis and Operator Theory, 2010, Valencia, O. Blasco, J. Bonet, J. Calabuig, D. Jornet (eds.), ContemporaryMath. 561 (2012), 3-47.

[11] Domanski, P., Langenbruch, M., Representation of multipliers on spaces ofreal analytic functions, Analysis (Munich) 32 (2012), 137-162.

[12] Erdélyi.A, et al., Tables of Integral Transforms I , McGraw-Hill ,New York, 1954

[13] Erdélyi.A, et al., Tables of Integral Transforms II ,McGraw-Hill ,New York, 1954

[14] G. Szegö, Orthogonal polynomials, rev. ed., Amer. Math. Soc. Colloq. Publ., vol. 23, Amer. Math. Soc, Providence, R. I., 1959. MR 21 \#5029.

[15] Hille.E, Analytic Function Theory, Vol. I. Ginn and Company, 1959.

[16] Hille.E, Analytic Function Theory, Vol.II . Ginn and Company, 1959.

[17] Van. Casteren, J.A ,Partial differential equations and operators , Part I ,http:/ bookboon.com

[18] Van. Casteren, J.A ,Partial differential equations and operators , Part II ,http/ bookboon.com

[19] Widder.D.V, The Laplace Transform. Princeton Mathematical Series, v. 6. Princeton UniversityPress, Princeton, N. J., 1941.

[20] Szegö,G Orthogonal polynomials, rev. ed., Amer. Math. Soc. Colloq. Publ., vol. 23, Amer. Math. Soc, Providence, R. I., 1959. MR 21 \#5029.

[21]Weeks W. T, Numerical inversion of the Laplace transform using Laguerre functions, J. Assoc.Comput. Mach., 13, 419-429, 1966. 